\title{
Peritoneal lavage examination as a prognostic tool in cases of gastric cancer
}

David Hoskovec ${ }^{1}$, Jozef Varga ${ }^{2}$, Petr Dytrych ${ }^{1}$, Ellen Konecna ${ }^{1}$, Jan Matek ${ }^{1}$

${ }^{1} 1^{\text {st }}$ Department of Surgery, General University Hospital, Prague, Czech Republic
${ }^{2}$ Department of Surgery, Hospital Bulovka, Prague, Czech Republic

Submitted: 20 December 2014

Accepted: 2 March 2015

Arch Med Sci 2017; 13, 3: 612-616

DOI: 10.5114/aoms.2016.64044

Copyright $\odot 2016$ Termedia \& Banach

\section{Abstract}

Introduction: Peritoneal tumor seeding is a common form of recurrence after gastric cancer surgery. The finding of free tumor cells and/or elevation of tumor markers in the peritoneal fluid could predict intraperitoneal tumor recurrence. The results of these examination can be used for indication of aggressive treatment modalities such as hyperthermic intraperitoneal chemotherapy (HIPEC).

Material and methods: We have operated on 105 patients suffering from gastric cancer. The control group consisted of 12 patients without malignant disease. Peritoneal lavage fluid or ascites was collected immediately after laparotomy and examined by cytology and biochemistry (levels of carcinoembryonic antigen (CEA) and Ca 19-9). Sensitivity, specificity, stage correlation and overall survival were observed.

Results: Elevation of tumor markers or the finding of free intraperitoneal tumor cells predicts recurrence. The prognosis of these patients is same as in stage IV TNM classification with median survival time less than 1 year $(p=0.713)$. Patients with negative cytology have median survival time 5 years contrary to them with positive cytology $(p<0.001)$. Sensitivity of the cytology was $34 \%$ and specificity was $85 \%$. Sensitivity of biochemistry was $53 \%$ (combination of both markers) and specificity was $100 \%$.

Conclusions: This study confirms the importance of peritoneal fluid examination for the prognosis. We cannot recommend routine use as an indicator for HIPEC due to low sensitivity, but the result of cytological examination is an independent factor for patient survival.

Key words: peritoneal cytology, peritoneal biochemistry, gastric cancer.

\section{Introduction}

Common features of gastric cancer are late diagnosis, unsatisfactory result of surgical treatment and poor effect of the oncological treatment.

Radical surgery is the only chance to treat gastric cancer patients. On the other hand, nearly $80 \%$ of patients are diagnosed in an advanced stage of the disease in the Czech Republic and radical surgery is impossible.

The aim of the surgery is RO resection. The peritoneal lavage examination is considered one way to exclude microscopic intraabdominal dissemination. The importance of cytology is well known, especially in cases of gastric and ovarian tumors. The positive peritoneal lavage cytology shifts the stage of the disease to stage IV according to the classification of the Japanese Research Society for Gastric Cancer (JRSGC) [1] and according to AJCC/UICC TNM classification version 7 [2].

\author{
Corresponding author: \\ David Hoskovec MD, PhD \\ $1^{\text {st }}$ Department of Surgery \\ General University Hospital \\ U nemocnice 2 \\ 12000 Prague 2 \\ Czech Republic \\ Phone: + 420224962259 \\ Fax: +420224963377 \\ E-mail: david.hoskovec@ \\ vfn.cz
}


We started examination of the intraabdominal fluid or peritoneal lavage in cases of gastric cancers to establish a rapid and precise method for diagnosis of microscopic spread of cancer cells inside the peritoneal cavity. The study was focused on identification of an early marker of peritoneal dissemination. We have planned to use this marker for indication of hyperthermic intraperitoneal chemotherapy in these tumors.

\section{Material and methods}

We have operated on 105 consecutive gastric cancer patients in the Department of Surgery, University Hospital Bulovka between 2003 and 2010. Inclusion criteria were gastric carcinoma confirmed by pathological examination before surgery. Exclusion criteria were liver and pulmonary metastases. There were 57 men and 48 women. Average age was 68 years ( 40 to 87 years) in men and 61 years (33 to 91 years) in women. The stages of the disease are summarized in Table I. There were 56 patients with diffuse type carcinoma and 45 patients with intestinal type carcinoma according to the Lauren classification. Tumor was totally dedifferentiated in 4 cases.

The type of radical surgery (50 patients in stage 0-III) depended on the Lauren classification and tumor localization. Total gastrectomy was performed in patient with diffuse carcinoma and with intestinal carcinoma with proximal localization (upper $2 / 3$ of the stomach). In cases of antral localization of intestinal carcinoma we performed subtotal gastrectomy $-4 / 5$ resection. Lymphadenectomy was in all cases D2 according to the Japanese Gastric Cancer Association. There was no positive resection margin in these patients (we checked it regularly during surgery) and surgery was always classified as R0 resection. Type of non-radical surgery in stage IV widely varied. We performed subtotal or total gastrectomy with D2 lymphadenectomy in cases without distant metastases (30 patients). In three cases the definitive pathological examination of the resection line was positive (despite negative peroperative frozen section examination). Palliative resection without lymphadenectomy was performed in $1 / 3$ of patients with distant metastases. We prefer resection in younger patients and in patients with a lower peritoneal cancer index according to Sugebaker. Surgery in the rest of the patient with metastases was only exploration (by laparotomy or laparoscopy) or gastroenteroanastomosis.
The control group consisted of 12 patients, 4 of whom were men and 8 women. Mean age was 47 years (36 to 58 years). All subjects in this group were operated on due to chronic cholecystitis confirmed by pathological examination report on the resected specimen. All these patients were examined by upper gastrointestinal (Gl) endoscopy in the preoperative period (maximally 10 weeks before surgery).

Samples of peritoneal fluid/lavage were taken immediately after laparotomy before any manipulation with the tumor and surrounding tissues. In cases where the ascites was present it was collected for examination. In other cases lavage of the epigastrium with $80 \mathrm{ml}$ of saline solution was performed. Samples were taken away $5 \mathrm{~min}$ later $(10 \mathrm{ml}$ for cytology and $5 \mathrm{ml}$ for biochemistry examination). The cytology specimens were centrifuged and the sediment was placed on the slide and examined after MGG (May-Grünwald-Giemsa) staining. The samples are evaluated for malignant cells by standard cytomorphological criteria by an experienced cytopathologist. Symptoms of malignancy were: presence of clusters, nuclear overlapping, increased nucleus to cytoplasm ratio and irregularity of nuclear membranes. The findings were assessed as benign, suspect and malignant. We counted suspect findings as positive too for the final results. Biochemistry evaluation was performed in the same manner as blood examination. We evaluated levels of CEA and Ca 19-9. Physiological levels were the same as in the blood (i.e. $0-4.6 \mu \mathrm{g} / \mathrm{l}$ for CEA and $0-37 \mathrm{U} / \mathrm{ml}$ for Ca 19-9).

The study was approved by Ethical Committee of the University Hospital Bulovka and all patients signed the informed consent.

Sensitivity and specificity tests were calculated from a $2 \times 2$ table.

We expected $100 \%$ prevalence of tumor cells in peritoneal fluid in cases of serosal infiltration or distant peritoneal metastases (49 patients). There were one patient in stage II, 10 patients in stage III and the rest in stage IV. We compared this group to the control group with chronic cholecystitis.

All patients were regularly checked in the postoperative period every 3 months in the first 2 years and after that every 6 months. There were clinical examinations in all patients (including patients with palliative surgery) and biochemistry, computed tomography (CT) or ultrasonography (US) and upper GI endoscopy in cases with radical operation. A medical oncologist indicated the type

Table I. Stage of disease

\begin{tabular}{|lcccccc|}
\hline Stage & 0 & I & II & III & IV & Total \\
\hline No. of patients. & 1 & 14 & 12 & 23 & 55 & 105 \\
\hline
\end{tabular}


of adjuvant (or palliative) chemotherapy. Adjuvant chemotherapy was used in patients with T3 and 4 tumors and/or positive lymph nodes. Radiotherapy was added in N3 patients younger than 65 years.

\section{Statistical analysis}

Survival of the patients was calculated according to Kaplan-Meier and group comparison was performed with the ANOVA test.

\section{Results}

\section{Sensitivity and specificity}

In the group of 49 patients with peritoneal infiltration there was a negative cytological finding in 33 cases. In fact there was a positive finding almost only in the subgroup of distant peritoneal metastases. The gastric wall infiltration subgroup (24 patients) had only 2 cases of suspicious cytology. In the control group there were 2 patients with a suspected positive cytological finding. So sensitivity for cytology is only $34 \%$ and specificity is $85 \%$. Peritoneal biochemistry was more sensitive. In our study CEA sensitivity was $38 \%$ and Ca $19-942 \%$. Combination of these markers achieved $53 \%$ because there was not coincident elevation of both observed markers (elevation of both markers together was found in 13 patients and 12 patients had elevation of either CEA or Ca 19-9). The elevation of CEA or Ca 19-9 was not found in any patients from the control group. Specificity of the biochemistry was 100\% (Tables II and III). The val- ue of intraperitoneal levels of CEA and Ca 19-9 did not match blood levels.

\section{Influence on staging and prognosis}

We found a positive cytologic finding in early stages of gastric cancer. There were positive findings in 3 of 14 patients in stage I. There was a similar result of examination in stages II and III (stage II three of 12 patients and stage III four of 23 patients). The highest percentage of positive cytology was in stage IV - nearly 40\% (Table IV).

The same findings were in biochemistry examination. Elevation of Ca 19-9 we observed in stages II and III and CEA elevation was found in stage III of the disease. There was not elevation of blood levels of both markers in these cases. Peritoneal biochemistry was always normal in all cases of stage 0 and I. There were huge difference between intraperitoneal and blood levels of CEA and Ca 19-9 in general (Table V).

The prognosis of patients with positive intraperitoneal cytology is worse than the negative cases (Figure 1). Median survival time was less than 1 year in patients with positive cytology (stage I-III). On the other hand, patients in stage 0-III and with negative peritoneal cytology had median survival time of 5 years. This difference in survival is statistically significant $(p<0.001)$. The same results were observed for survival according to stage grouping. Prognosis of cytologically positive patients is the same as the prognosis of patients in stage IV of the disease regardless of other parameters of TNM staging $(p=0.713)$.

Table II. Cytology - sensitivity $(N=49)$

\begin{tabular}{|lccc|}
\hline Peritoneal cytology & \multicolumn{2}{c|}{ Peritoneal infiltration } & Total \\
\cline { 2 - 4 } & Local (gastric wall) & Distant & 33 \\
\hline Benign & 20 & 13 & 14 \\
\hline Suspicious & 4 & 10 & 2 \\
\hline Malignant & $24 \mathrm{pts}$ & 2 & 49 \\
\hline Total & $25 \mathrm{pts}$ & \\
\hline
\end{tabular}

Table III. Biochemistry - sensitivity $(N=48)$

\begin{tabular}{|lccc|}
\hline Variability & \multicolumn{2}{c|}{ Peritoneal infiltration } & Total \\
\cline { 2 - 4 } & Local (gastric wall) & Distant & 30 \\
\hline CEA normal & 21 & 9 & 18 \\
\hline CEA elevation & 2 & 16 & 28 \\
\hline Ca 19-9 normal & 18 & 10 & 20 \\
\hline Ca 19-9 elevation & 5 & 15 & 25 \\
\hline CEA and/or Ca 19-9 elevation & 6 & 19 & \\
\hline
\end{tabular}

Levels of oncomarkers were not examined in 1 patient due to health insurance policy limitation. 
Table IV. Cytological finding according to stage

\begin{tabular}{|lcccc|}
\hline Stage & \multicolumn{3}{c|}{ Cytology } \\
\cline { 2 - 4 } & Benign & Suspicious & Malignant & Total \\
\hline 0 & 1 & 3 & 1 \\
\hline I & 11 & 2 & 14 \\
\hline II & 10 & 4 & 3 & 23 \\
\hline III & 19 & 18 & 3 & 12 \\
\hline IV & 34 & 27 & 3 & 105 \\
\hline Total & 75 & & & 23 \\
\hline
\end{tabular}

\section{Disscusion}

The malignant transformation of the cells changes expression of the surface proteins and causes the release of these cells into the peritoneal cavity [3]. It is probably the first stage of peritoneal carcinomatosis. The finding of the free tumor cells corresponds with $T$ stage of the disease. Serosal involvement of the tumor can cause peritoneal seeding. However, a tumor without serosal invasion can also release free cancer cells into the peritoneal cavity [4]. Vascular invasion and lymph metastasis are other factors resulting in peritoneal cancer seeding [5].

Positive peritoneal cytology predicts poor prognosis of gastric tumors.

Intraperitoneal levels of the oncomarkers have similar prognostic value as peritoneal cytology. The most frequently used marker is CEA. But there are fewer reports focusing on this problem in comparison to reports about cytology.

The mechanism of CEA release into the peritoneal cavity is unclear. Tumor cells infiltrating the serosal layer can release CEA. However, there are data about the presence of CEA in serosal negative tumors [6]. Some authors use other markers such as Ca 19-9 and CA 125 [7-12]. The cut-off value for CEA is $100 \mathrm{ng} / \mathrm{g}$ of total protein or $1 \mathrm{ng} / \mathrm{ml}[6,13]$.

Peritoneal lavage biochemistry has better sensitivity and specificity than cytology according to some authors [13]. Nearly all data about peritoneal biochemistry were captured in patients with gastric tumors. Possible implication for other upper GI tumors is usually not monitored.

Most studies dealing with free cancer cells are focused on gastric tumors. The majority of these studies are from Asian countries. Reports from Europe and the US are less common. As mentioned before, a positive cytological finding in the peritoneal fluid means an advanced disease (stage IV). The finding of free cancer cells in the peritoneal cavity correlates with size of the tumor, number of positive lymph nodes and involvement of the lymphatic and blood vessels, serosal involvement and with distant metastasis.
Table V. Average levels of CEA and Ca 19-9 in serum and in the peritoneal cavity

\begin{tabular}{|lcc|}
\hline Serum & CEA & $150(0-47.7) \mu \mathrm{g} / \mathrm{l}$ \\
\cline { 2 - 3 } & Ca $19-9$ & $185(0-1960) \mathrm{U} / \mathrm{ml}$ \\
\hline Peritoneal cavity & CEA & $233(0-6000) \mu \mathrm{g} / \mathrm{l}$ \\
\cline { 2 - 3 } & Ca $19-9$ & $5768(0-12000) \mathrm{U} / \mathrm{ml}$ \\
\hline
\end{tabular}

All reports confirm worsening of the patient's prognosis with positive cytology despite various detection rates [14-17]. A consequence of these findings is the new TNM classification. However, not all studies have confirmed the value of this classification for prognosis [18].

Positive peritoneal cytology could predict the site of recurrence. It is an independent prognostic factor of intraperitoneal dissemination and recurrence of the disease (sensitivity $56 \%$, specificity 97\%) $[19,20]$.

The results of the biochemistry examination are not so evident. For example, Li et al. in their

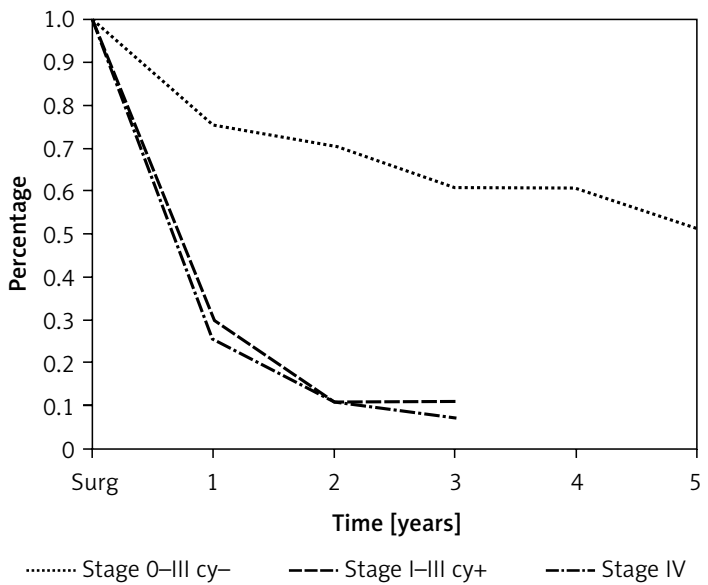

\begin{tabular}{|l|c|c|c|c|c|c|}
\hline \multicolumn{7}{|l|}{ No. of patients at risk } \\
\hline 0-III cy- & 41 & 31 & 29 & 25 & 25 & 21 \\
\hline I-III cy+ & 9 & 3 & 1 & 1 & & \\
\hline IV & 55 & 14 & 6 & 4 & & \\
\hline
\end{tabular}

Figure 1. Overall survival 
paper showed that the level of CEA has a higher prognostic importance for peritoneal seeding than cytology [14]. On the other hand, Bold et al. did not confirm those results but in his paper peritoneal dissemination was predicted according to intraperitoneal level of CA 125 (cut-off value $35 \mathrm{IU} / \mathrm{ml})[21]$.

In conclusion, our results indicate the importance of the peritoneal lavage examination. This examination could indicate the advanced disease sooner than other techniques. However, it is necessary to improve sensitivity and specificity of the examination. Proper identification of the malignant cells before or during surgery (due to manipulation of the tumor) indicates that conventional surgery cannot achieve the RO resection. There is a space for an enhanced oncological approach either during surgery (for example hyperthermic intraperitoneal cytostatic lavage) or postoperatively.

We performed this study before implementation of the TNM version 7. Our results confirmed the justifiability of the new version of IUCC/TNM and the importance of peritoneal fluid examination in non-Asian countries.

\section{Acknowledgments}

Prvouk 8.2 Charles University Prague RVOVFN64165.

\section{Conflict of interest}

The authors declare no conflict of interest.

\section{References}

1. Aiko T, Sasako M. The new Japanese clasification of gastric carcinoma: points to be revised. Gastric Cancer 1998; $1: 25-30$

2. Washington K. $7^{\text {th }}$ Edition of the AJCC Cancer Staging Manual: Stomach. Ann Surg Oncol 2010; 17: 3077-9.

3. Makary MA, Warshaw AL, Centeno BA, Willett CG, Rattner DW, del Castillo CF. Implications of peritoneal cytology for pancreatic cancer management. Arch Surg 1998; 133: 361-5.

4. Marutsuka T, Shimda S, Shiomori K, Ogawa M. Peritoneal metastasis after operation for non-serosa-invasive gastric carcinoma and prophylatic strategy for peritoneal dissemination. International Congress Series 2003; 1255: 103-7.

5. Swan R, Miner TJ. Current role of surgical theray in gastric cancer. World J Gastroenterol 2006; 12: 372-9.

6. Nishiyama M, Takashima I, Tanaka T, et al. Carcinoembryonic antigen levels in the peritoneal cavity: useful guide to peritoneal recurrence and prognosis for gastric cancer. World J Surg 1995; 19: 133-7.

7. Hayes N, Wayman J, Wadehra V, Scott DJ, Raimes SA, Griffin SM. Peritoneal cytology in the surgical evaluation of gastric carcinoma. Br J Cancer 1999; 79: 520-4.

8. Vogel P, Rüschoff J, Kümmel $S$, et al. Prognostic value of microscopic peritoneal dissemination. Dis Colon Rectum 2000; 43: 92-100.
9. Wang JY, Lin SR, Lu CY, et al. Gastric cancer cell detection in peritoneal lavage: RT-PCR for carcinoembryonic antigen transcripts versus combined cytology with peritoneal carcinoembryonic antigen levels. Cancer Letters 2005; 223: 129-35.

10. Wang Z, Hao B, Yang Y, Wang R, Li Y, Wu Q. Prognostic role of SPARC expression in gastric cancer: a meta-analysis. Arch Med Sci 2014; 10: 863-9.

11. Wang JY, Lin SR, Lu CY, et al. Gastric cancer cell detection in peritoneal lavage: RT-PCR for carcinoembryonic antigen transcripts versus combined cytology with peritoneal carcinoembryonic antigen levels. Cancer Lett 2005; 223: 129-35.

12. Zhao K, Zhu BS, Gong W, et al. SN50 enhances the effects of LY294002 on cell death induction in gastric cancer cell line SGC7901. Arch Med Sci 2013; 9: 990-8.

13. Yu X, Luo L, Wu Y, et al. Gastric juice miR-129 as a potential biomarker for screening gastric cancer. Med Oncol 2013; 30: 365.

14. Li JK, Zheng M, Miao CW, Zhang JH, Ding GH, Wu WS, Peritoneal lavage cytology and carcinoembryonic antigen determination in predicting peritoneal metastasis and prognosis of gastric cancer. World I Gastroenterol 2005; 46: 7374-7.

15. Majima T, Ichikura T, Mochizuki H. Prognostic significance of the cytologic features of free cancer cells in the peritoneal cavity of patints with gastric cancer. Surg Today 2002; 32: 35-9.

16. de Manzoni G, Verlato G, di Leo A, et al. Peritoneal cytology does not increase the prognostic information provided by TNM in gastric cancer. World I Surg 2006; 30: 579-84.

17. Ribeiro Jr U, Gama-Rodrigues JJ, Safatle-Ribeiro AV, et al. Prognostic significance of intraperitoneal free cancer cells obtained by laparoscopic peritoneal lavage in patients with gastric cancer. J Gastrointest Surg 1998; 2: 244-9.

18. Sugarbaker PH. Adjuvant intraperitoneal chemotherapy for advanced primary gastric cancer. Scand I Surg 2006; 95: 270-3.

19. Graziosi L, Marino E, Cavazzoni E, Donini A. Prognostic value of the seventh AJCC/UICC TNM classification of non-cardia gastric cancer. World J Surg Oncol 2013; 11: 103.

20. Bando E, Yonemura Y, Takeshita Y, et al. Intraoperative lavage for cytological examination in 1297 patients with gastric carcinoma. Am J Surg 1999; 178: 256-62.

21. Bold R, Ota DM, Ajani JA, Mansfield PF. Peritoneal and serum tumor markers predict recurrence and survival of patients with resectable gastric cancer. Gastric Cancer 1999; 2: 1-7. 